\title{
Glass Bottle
}

National Cancer Institute

\section{Source}

National Cancer Institute. Glass Bottle. NCI Thesaurus. Code C43172.

A glass vessel with a narrow neck designed to accept a specific closure. 